NASA Technical Memorandum 107607

\title{
A CONSISTENT-MODE INDICATOR FOR THE EIGENSYSTEM REALIZATION ALGORITHM
}

Richard S. Pappa, Kenny B. Elliott, and Axel Schenk

April 1992

\section{N $15 \wedge$ \\ National Aeronautics and \\ Space Administration \\ Langley Research Center \\ Hampton, Virginia 23665}

(NASA-TM-107507) A CONSISTENT-MODE 
$=-\infty$ 


\section{A CONSISTENT-MODE INDICATOR FOR THE EIGENSYSTEM REALIZATION ALGORITHM}

\author{
R. S. Pappa and K. B. Elliou \\ NASA Langley Research Center \\ Hampton, Virginia
}

\author{
A. Schenk \\ Gcrman Aerospace Research Establishment (DLR) \\ Gruingen, Germany
}

\begin{abstract}
A new method is described for assessing the consistency of structural modal parameters identified with the Eigensystem Realization Algorithm. Identification results show varying consistency in practice duc to many sources including high modal density, nonlincarity, and inadequate excitation. Consistency is considered to be a reliable indicator of accuracy. The new method is the culmination of many ycars of experience in developing a practical implementation of the Eigensystem Realization Algorithm. The effectiveness of the method is illustrated using data from NASA Langley's Controls-StructuresInteraction Evolutionary Model.
\end{abstract}

\section{Introduction}

The dynamic behavior of most acrospace structures is adequately described using modal parameters (natural frequencies, mode shapes, damping factors, and modal masses). The objective of structural modal identification is to obtain a valid modal representation over a specified frequency range for all spatial degrees-of-freedom. This objective is considerably different than identifying an input-output map only at particular degrees-of-freedom where control actuators and sensors are located. ${ }^{1} \mathrm{~A}$ full spatial modal representation permits several tasks to be performed which cannot be performed using an inputoutput map derived for control purposes. These tasks include validation of structural modeling procedures and assumptions, prediction of systcm dynamics using modal parameters of individual components, investigation of more-effective actuator and sensor locations for control purposes, and improved characterization of disturbances occurring at unexpected locations on a spacecraft during operation.

It is relatively straightforward to cstimate structural modal parameters experimentally using a varicty of available approaches. 2,3 However, it is generally much more difficult to establish reliable confidence values for cach result. Confidence criteria based on noise characteristics are available ${ }^{4}$ but are of limited usefulness in practical applications. In modal-survey tests, identification difficulties arise primarily from high modal density, nonlinearity, weakly excited modes, local modes, nonstationarities, rattling, ctc., not from instrumentation noisc. The simultaneous effects of these conditions are in general impossible to include explicitly in confidence calculations.

The Consistent-Mode Indicator (CMI) described in this paper provides a reliable, relative measure of accuracy for structural modal parameters identified with the Eigensystem Realization Algorithm (ERA). ${ }^{5-7}$ A single value ranging from 0 to 100 percent is obtained for each identificd mode. Furthermore, the results can be decomposed into constituent components associated with each inpul (initial condition) and output (response mcasurement), or input-output pair. Both temporal and spatial consistency calculations are included in the formulation. Modes with $\mathrm{CMI}$ values greater than approximatcly 80 percent are identified with high confidence. Modes with values ranging from 80 to 1 percent display moderate to large uncertainty. Fictitious "computational modes" have CMI values of zero.

The first part of this paper contains a brief summary of ERA followed by a complete description of CMI. The second part illustrates the concepts using recent laboratory data from NASA Langley's Controls-Structures-Interaction (CSI) Evolutionary Model (CEM). ${ }^{8}$ The CEM is a large flexible rescarch structure being used to experimentally assess the level of confidence with which CSI technolcgy can be applied to future spacecraft.

\section{The Eigensystem Realization Algorithm}

A linite-dimensional, linear, time-invariant dynamic system can be represented by the state-variable equations:

$$
\begin{aligned}
& \dot{x}(t)=A_{c} x(t)+B u(t) \\
& y(t)=C x(t)
\end{aligned}
$$

where $\mathrm{x}$ is an $\mathrm{n}$-dimensional state vector, $\mathbf{u}$ is a $\mathbf{p}$ dimensional excitation vector, and $y$ is a q-dimensional response vector. A special solution to these equations is the impulse response function: 


$$
Y(t)=C e^{A_{b} \cdot B}
$$

for $t \geq 0$. The ith column of $Y(t)$ contains the free response of the system, with $x(0)=0$, to a unit-impulse excitation applied at the ith input location at $\mathrm{t}=0$.

With sampled data, this solution can be expressed as

$$
Y(k)=C A^{k} B
$$

for $k \geq 0 . A=e^{A_{c} A t}$ is the statc-transition matrix and $\Delta t$ is the data sampling interval.

The problem of system realization is as follows: Given a sequence of experimentally measured matrices $Y(k)$, for $k=0,1,2, \ldots$, construct a triplet $[A, B, C]$ such that the above relationship is satisfied as closely as possible. Note that $[A, B, C]$ is not unique since the set $\left[T^{-1} A T, T^{-1} B, C T\right]$, for any nonsingular matrix $T$, also satisfies the state-variable cquations.

The ERA solution to the system realization problem uses singular value decomposition of the generalized Hankel matrix:

$$
H(0)=U \Sigma V^{T}
$$

Matrix $\mathbf{H}(0)$ consists of time-shifted $\mathrm{Y}(\mathbf{k})$ submatrices. In practice, cvery block row and block column of $H(0)$ normally contains data shifted in time by one sample from data in the previous block row or block column. The only exceptions are the final block row and column which are shifted by a larger number of time samples (by default, 10 samples) in order to calculate EMAC, discussed later in the paper. Also, in large modal surveys involving hundreds of response measurements, a fraction of the rows of $H(0)$ below row $q(q=$ no. of measurements) may generally bc deleted without loss of accuracy duc to the large redundancy of information.

Retaining the $\mathrm{n}$ largest singular values, an nth-order realization is computed as follows:

$$
\begin{aligned}
& A=\Sigma_{n}{ }^{-1 / 2} U_{n}{ }^{T} H(1) V_{n} \Sigma_{n}{ }^{-1 / 2} \\
& B=\Sigma_{n}{ }^{1 / 2} V_{n}{ }^{T} E_{p} \\
& C=E_{q}{ }^{T} U_{n} \Sigma_{n}^{1 / 2}
\end{aligned}
$$

where $H(1)$ is a matrix of the same form as $H(0)$ but whose data are shifted in time by one additional sample. $E_{p}$ and $E_{q}$ are pth-order and qth-order selector matrices.
This realization is transformed to modal coordinates using the eigenvalues $\mathrm{Z}$ and eigenvector matrix $\Psi$ of $\mathrm{A}$ :

$$
\begin{aligned}
& A^{\prime}=\Psi^{-1} A \Psi=Z \text { (diagonal) } \\
& B^{\prime}=\Psi^{-1} B \\
& C^{\prime}=C \Psi
\end{aligned}
$$

The modal damping rates $\sigma_{i}$ and damped natural frequencies $\omega_{i}$ are the real and imaginary parts of the eigenvalues after transformation back to the continuous domain:

$$
s_{i}=\sigma_{i} \pm j \omega_{i}=\ln \left(z_{i}\right) / \Delta t
$$

Modal participation factors and mode shapes are the corresponding rows of $B^{\prime}$ and columns of $C^{\prime}$, respectively.

In practice, some modal parameters obtained using this approach are inaccurate due to high modal density, nonlinearity, etc. CMI is used to assess the relative accuracy of the various results.

\section{Consistent-Mode Indicator}

The Consistent-Mode Indicator, CMI, is computed for mode $i$ as the product of two other parameters, EMAC and MPC:

$$
\mathrm{CMI}_{\mathrm{i}}=\mathrm{EMAC}_{\mathrm{i}} \cdot \mathrm{MPC}_{\mathrm{i}} \quad(\mathrm{x} 100 \%)
$$

The Extended Modal Amplitude Coherence, EMAC, quantifies the temporal consistency of the identification results. The Modal Phase Collinearity, MPC, quantifics the spatial consistency of the identification results. Practical experience has shown that both conditions must be satisfied simultaneously to ensure accurate results. These two parameters are described separatcly in the following sections.

\section{Extended Modal Amplitude Coherence}

The Extended Modal Amplitude Coherence, EMAC, is . computed using the identified modal parameters. Mode shape components for data at $t=0$ are compared with corresponding components for data at a time instant $t=T_{0}$ (for outputs) or $t=T_{1}$ (for inputs) stored in the final block row and final block column, respectively, of the ERA generalized Hankel matrices. An EMAC value is computed for each of the $p$ inputs (initial conditions) and $q$ outputs (response measurements), for every mode. 
Let $\left(\phi_{i j}\right)_{0}$ be the identified mode shape component for mode $i$ and response measurement $j$ at $t=0$ and $\left(\phi_{i j}\right)_{T_{0}}$ be the corresponding identified component at $t=T_{0}$. The identified eigenvalue for mode $i$ is $s_{i}$. Compute a predicted value of $\left(\phi_{i j}\right)_{T_{o}}$ as follows:

$$
\left(\tilde{\phi}_{i j}\right)_{T_{o}}=\left(\phi_{i j}\right)_{0} \cdot e^{\mathbf{s}_{1} T_{o}}
$$

Temporal consistency is quantified by comparing $\left(\phi_{i j}\right)_{T_{o}}$ and $\left(\tilde{\phi}_{i j}\right)_{T_{0}}$. The actual and predicted magnitudes ane compared using the ratio of the magnitudes:

$$
\begin{aligned}
\mathbf{R}_{\mathrm{ij}} & =\left|\left(\phi_{\mathrm{ij}}\right)_{\mathrm{T}_{\mathrm{o}}}\right| /\left(\tilde{\phi}_{\mathrm{ij}}\right)_{\mathrm{T}_{\mathrm{o}}} \mid \text { for }\left|\left(\phi_{\mathrm{ij}}\right)_{\mathrm{T}_{\mathrm{o}}}\right| \leq\left|\left(\tilde{\phi}_{\mathrm{ij}}\right)_{\mathrm{T}_{\mathrm{o}}}\right| \\
& =\left|\left(\tilde{\phi}_{\mathrm{ij}}\right)_{\mathrm{T}_{\mathrm{o}}}\right| /\left(\phi_{\mathrm{ij}}\right)_{\mathrm{T}_{\mathrm{o}}} \mid \text { otherwisc. }
\end{aligned}
$$

The actual and predicted phase angles are also compared. Lelling $P_{i j}=\operatorname{Arg}\left(\left(\phi_{i j}\right)_{T_{o}} /\left(\bar{\phi}_{i j}\right)_{T_{0}}\right), \quad-\pi \leq P_{i j} \leq \pi, \quad a$ wcighting factor is determined as follows:

$$
\begin{aligned}
W_{i j} & =1.0-\left(\left|P_{i j}\right| /(\pi / 4)\right) \text { for }\left|P_{i j}\right| \leq \pi / 4 \\
& =0.0 \text { otherwisc }
\end{aligned}
$$

All Output EMAC for mode $i$ and response measurement $\mathrm{j}$ is then computed as:

$$
\operatorname{EMAC}^{0}{ }_{\mathrm{ij}}=\mathrm{R}_{\mathrm{ij}} \cdot \mathrm{W}_{\mathrm{ij}} \quad(\times 100 \%)
$$

An Input EMAC for mode $i$ and initial condition $k$, EMAC $^{l}$ ik, is similarly computed using the identificd modal participation factors.

Using these results, an EMAC value is associated with every $j-k$ 'th input-output pair as follows:

$$
\text { EMAC }_{\mathrm{ijk}}=\mathrm{EMAC}_{\mathrm{ij}}{ } \cdot \mathrm{EMAC}^{\mathrm{I}} \mathrm{ik} \quad(\times 100 \%) .
$$

Finally, to condense all EMAC results for mode $i$ into a single value, a weighted average of the individual results is computed:

$$
\begin{aligned}
\operatorname{EMAC}_{i} & =\frac{\sum_{j=1}^{q} \sum_{k=1}^{p} \operatorname{EMAC}_{i j k} \cdot\left|\phi_{i j}\right|^{2} \cdot\left|\phi_{i k}\right|^{2}}{\sum_{j=1}^{q} \sum_{k=1}^{p}\left|\phi_{i j}\right|^{2} \cdot\left|\phi_{i k}\right|^{2}} \\
& =\frac{\left(\sum_{j=1}^{q} \operatorname{EMAC}_{i j} \cdot\left|\phi_{i j}\right|^{2}\right)\left(\sum_{k=1}^{p} \operatorname{EMAC}_{i k}^{\prime} \cdot\left|\phi_{i k}\right|^{2}\right)}{\sum_{j=1}^{q}\left|\phi_{i j}\right|^{2} \cdot \sum_{k=1}^{p}\left|\phi_{i k}\right|^{2}}
\end{aligned}
$$

where $\phi_{i j}$ and $\phi_{i k}$ are mode shape and modal participation components, respectively. A weighting factor of $|\phi|^{2}$ is used to achieve an energy-type emphasis.

In the original formulation of ERA ${ }^{5}$, a parameter known as the Modal Amplitude Coherence $(\gamma)$ was introduced. EMAC is a much-improved version of this formulation. Under certain common conditions, $\gamma$ values can be high (cven 100 percent) for all eigenvalues. This insensitivity is avoided with EMAC. The term "extended" in the new name refers to the extension of the primary data analysis window for the final block row and column of the generalized Hankel matrix. EMAC quantifies the consistency of the identified modal parameters by mcasuring the predictability of the results in this extended time interval. As discussed in the Introduction, this test is much more difficult to fulfill than testing only the predictability of the results in the primary analysis window, which is what $\gamma$ does. EMAC also involves many fewer calculations than $\gamma$. Most importantly, however, it provides a more-sensitive, more-reliable approach with proven usefulness based on many successful applications.

\section{Modal Phase Collinearity}

Modal Phase Collinearity, MPC, quantifies the spatial consistency of the identification results. For classical normal modes, all locations on the structure vibrate exactly in-phase or out-of-phase with one another; i.e., the corresponding mode shape is a rcal or "monophase" vector.

With monophase behavior, the variance-covariance matrix of the real and imaginary parts of the mode shape vectors has only onc nonzero eigenvalue. If the identified modeshape phase angles are uncorrelated, on the other hand, the two cigenvalues of this matrix will be approximately equal. MPC quantifies the degree of monophase behavior by comparing the relative size of the largest and smallest cigenvalues of the variance-covariance matrix.

Let $\Phi_{i}^{\prime}$ and $\Phi_{i}^{\prime \prime}$ be the real and imaginary parts, respectively, of the identified mode shape for mode i. Calculate the variance and covariance of the real and imaginary parts:

$$
\begin{aligned}
& S_{x x}=\Phi_{i}^{\prime T} \Phi_{i}^{\prime} \\
& S_{y y}=\Phi_{i}^{\prime \prime} \Phi_{i}^{\prime \prime} \\
& S_{x y}=\Phi_{i}^{\prime} \Phi_{i}^{\prime \prime}
\end{aligned}
$$


Letting

$$
\begin{gathered}
\mu=\frac{S_{y y}-S_{x x}}{2 S_{x y}} \\
\beta=\mu+\operatorname{sgn}\left(S_{x y}\right) \sqrt{\mu^{2}+1} \\
\tau=\tan ^{-1} \beta,
\end{gathered}
$$

the eigenvalues of the variance-covariance matrix are:

$$
\begin{aligned}
& \lambda_{1}=S_{x x}+\frac{S_{x y}\left(2\left(\mu^{2}+1\right) \sin ^{2} \tau-1\right)}{\mu} \\
& \lambda_{2}=S_{y y}-\frac{S_{x y}\left(2\left(\mu^{2}+1\right) \sin ^{2} \tau-1\right)}{\mu}
\end{aligned}
$$

MPC for mode $i$ is then defined as follows:

$$
\mathrm{MPC}_{i}=\left[2 \cdot\left(\frac{\lambda_{1}}{\lambda_{1}+\lambda_{2}}-0.5\right)\right]^{2}(\times 100 \%)
$$

$\mathrm{MPC}_{\mathrm{i}}$ values range from 0 for a mode with completely uncorrelated phase angles to 100 percent for a monophase result.

The formulation discussed above is based on the original definition of Modal Phase Collinearity $(\mu)^{5}$, except normalized to generate values ranging from 0 to 100 percent. The smallest possible value of $\mu$ was inadvertently limited to 25 percent. Also, the following two practical extensions of the MPC concept are presented for the first time:

\section{Unwelghted MPC}

The definition of MPC described above provides a natural weighting based on the magnitude of the individual modeshape components. This is desirable because phase angle results for small experimentally determined mode shape components are often inaccurate due to measurement limitations. However, it is also useful to repeat the calculations without this natural weighting imposed; i.e., by normalizing each mode shape component to unit magnitude before calculating the variance and covariance values. For global modes, this so-called unweighted MPC will be approximately equal to the standard weighted MPC discussed above. However, for local modes, the unweighted value will be substantially smaller than the weighted value. The magnitude of the difference provides a quick and effective indicator of global versus local response behavior.

\section{Phase Rotation for Free-Decay Data}

When ERA is applied to (displacement/force or acceleration/force) impulse response functions, the identified mode shapes display the classical characteristic of large imaginary parts and small real parts. With free-decay data corresponding to arbitrary initial conditions, however, the identified mode shapes have arbitrary mean phase angle. Before these shapes can be plotted as geometric deformations or used in certain subsequent calculations such as the Phase Resonance Criterion ${ }^{9}$, they must be rotated to align best with $\pm 90^{\circ}$. The necessary rotation angle $\alpha$ is determined during the MPC calculation:

$$
\alpha=\tau+\pi / 2
$$

\section{Application Example}

\section{Test Article and Modal Survey}

Fig. 1 shows the Controls-Structures-Interaction (CSI) Evolutionary Model (CEM) ${ }^{8}$ located at the NASA Langley Research Center. The structure is part of a testbed used to develop CSI ground test methods. It has been designed to possess the dynamic properties of a typical future large spacecraft. These properties include low frequency structural modes, modal clusters, local appendage dynamics, and both high and low levels of damping.

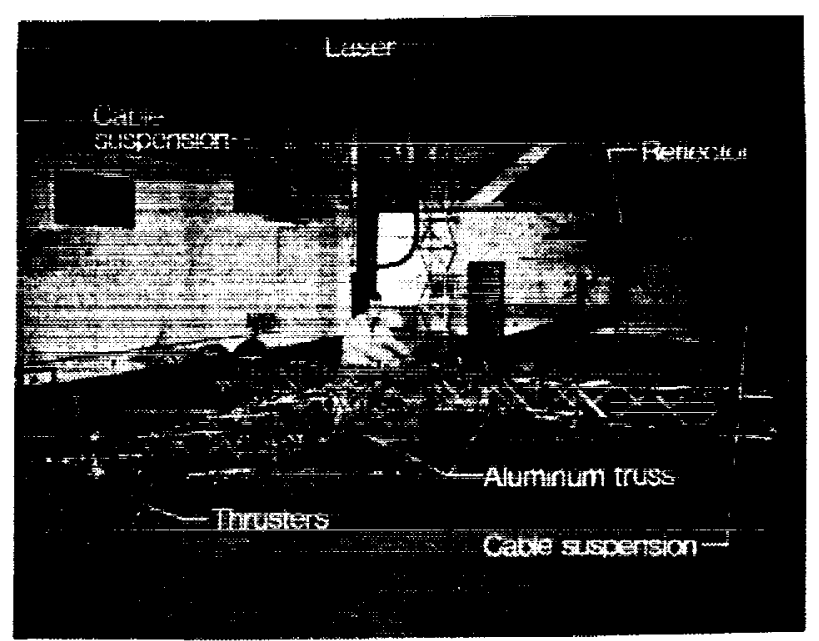

Fig. 1 CSI Evolutionary Model

The CEM consists of a 55 foot long truss with several appendages which possess varying degrees of flexibility. The truss is constructed of aluminum tubes assembled into 10 inch cubical bays. The structure is suspended by two cables attached to the laboratory ceiling through isolation springs. By using soft springs in series with long suspension cables, the six "pseudo-rigid body" modes have 
frequencics below $1 \mathrm{~Hz}$. The first flexible mode occurs at $1.5 \mathrm{~Hz}$ with approximately 30 modes occurring below 10 $\mathrm{Hz}$. For modal identification experiments, the CEM was instrumented with 195 piczo-film accelerometers and 18 servo accelerometers. Excitation was supplicd through 16 on-board cold-gas thrusters operating in pairs at 8 locations. The thrusters produce up to $2.2 \mathrm{lbs}$ of force over a bandwidth of approximatcly $4.5 \mathrm{~Hz}$.

A modal test was performed using all 213 accelerometers and 8 thruster pairs in a multi-input, multi-output (MIMO) test configuration. Uncorrelated shaped random noise signals were applicd simultancously to all exciters. A total of $1704(8 \times 213)$ frequency response functions (FRFs) with 2048 lines of resolution from 0 to $50 \mathrm{H} /$ were generated. The FRFs were computed using a commercial MIMO FRF calculation procedure ${ }^{10}$ with 100 averages to minimize noise effects. Frequency lines from 0 to $6.25 \mathrm{~Hz}$ were extracted and inverse Fourier transformed to obtain impulse response functions for the ERA analyses discussed below.

Before performing the ERA analysis, it is useful to calculate the average power spectrum (APS) and mode indicator function (MIF) ${ }^{11}$ direclly from the FRF data. These functions are defined as follows:

$$
\begin{gathered}
\operatorname{APS}(\zeta)=\frac{\sum_{i=1}^{N}\left|H_{i}(f)\right|^{2}}{N} \\
\operatorname{MIF}(\Gamma)=\left[1.0-\frac{\sum_{i=1}^{N}\left|H_{i}^{\prime}(f)\right| H_{i}(f) \mid}{\sum_{i=1}^{N}\left|H_{i}(I)\right|^{2}}\right] \cdot 1000
\end{gathered}
$$

where $H_{i}{ }^{\prime}(f)$ and $\left|H_{i}(f)\right|$ are the real part and magnitude, respectively, of the ith FRF. $N$ is the total number of FRFs included in the calculation. Both of these functions display peaks at cach natural frequency. Additionally, the APS shows the relative magnitude of cach modal response. The MIF provides no information concerning modal response magnitudes; however, the resolution of individual modes is much higher.

The APS and MIF calculated using all 1704 FRFs arc shown in Fig. 2. It can be stated with certainty based on these results that at least 16 modes occur between 0 and $6.25 \mathrm{~Hz}$. However, because of the complexity of the struclure and indicated by NASTRAN analytical predictions $^{8}$, some of the observed peaks probably represent more than onc mode. As an example, two pscudo-rigid body modes are known to occur between 0.7 and $0.8 \mathrm{H} z$ although this fact cannot be deduced from either function. Also note the wide variation of modal response magnitudes indicated by APS peaks ranging from 100 to Icss than 1. Modes with low response magnitudes such as those ncar 0.2 and $3.4 \mathrm{~Hz}$ are much more difficult to identify accurately than modes with high response magniludes.
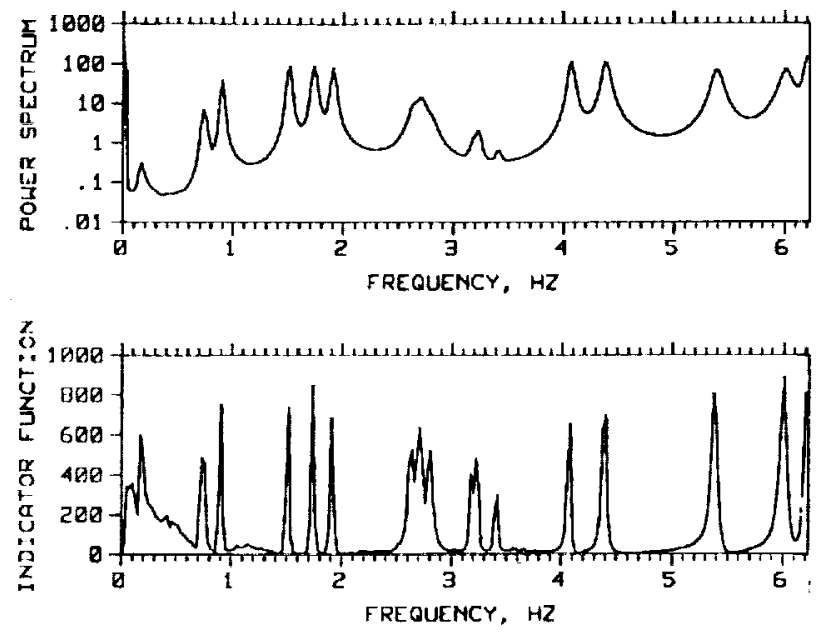

Fig. 2 Average Power Spectrum and Mode Indicator Function

\section{Ldenification Results}

Previous experience has shown that significant changes can occur in ERA analyses as a function of the assumed number of modes. In particular, optimum accuracy for different modes typically occurs at different numbers of assumed modes. Also, wcakly excited modes often require rclatively high numbers of assumed modes to be properly identificd. For these reasons, the assumed number of modes is incremented over a wide range of values in most applications. Based on the estimated number of modes from Fig. 2, the assumed number of modes is incremented for the CEM data analysis from 2 up to 60 (in steps of 2).

The identified natural frequencies as a function of the assumed number of modes are plotted in Fig. 3. These results were generated using all 1704 FRFs simultancously in an $8 \times 213 \mathrm{MIMO}$ analysis. Each row of results corresponds to a separate ERA analysis with a specifical number of assumed modes. Each detected mode is represented by a vertical dash at the associated frequency. The confidence in cach result is expressed by the length of the vertical dash which is proportional to CMI. The highest confidence $(\mathrm{CMI}=100 \%)$ is attained if the distance between minor tic marks on the vertical axis is filled. Eigenvalues with low CMI (less than 5 percent) are excluded from the figure. 
A wide spread of $\mathrm{CMI}$ values is indicated by the presence of both solid and dashed or dotled lines. Several modes with low CMI values occur in the frequency interval from 2.6 to $3.3 \mathrm{~Hz}$. These modes are known to be associated primarily with the flexible reflector and are known to occur in clusters. No thrusters are located directly on the reflector surface so that these modes cannot in general be excited individually. The result is a reduction of controllability and a corresponding decrease in both temporal and spatial consistency. Although engineering judgement is required to determine the exact cause of low CMI values, low values reliably indicate those results which should not be accepted verbatim as accurate modal parameters. As illustrated later in this paper, results for particular modes can often be improved without performing additional tests once this initial CMI information is available. Of course, additional tests can also be performed once certain areas of difficulty are known.

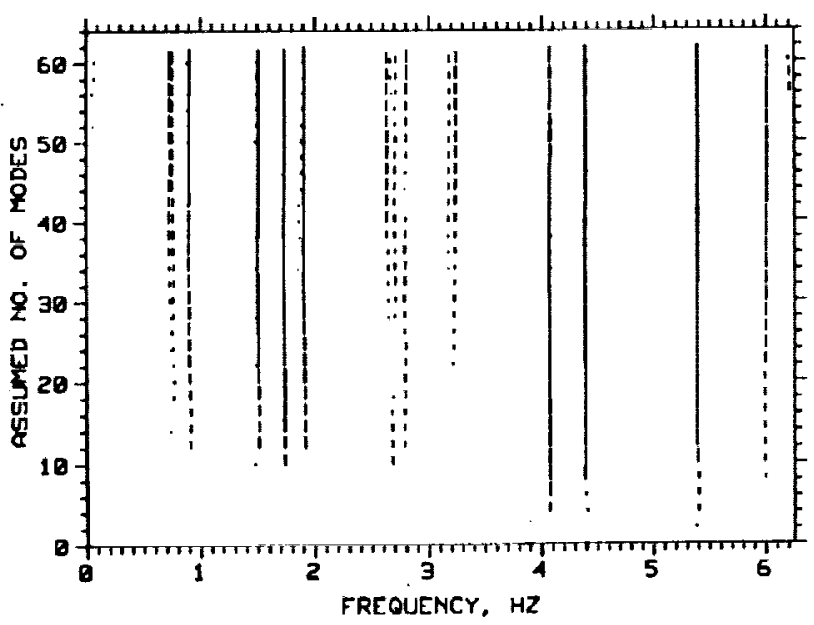

Fig. 3 Identified Natural Frequencies (Lengths of Vertical Dashes Proportional to CMI)

Fig. 4 shows an expanded view of the results in a narrow frequency interval ncar $0.9 \mathrm{~Hz}$. Corresponding damping. EMAC, and MPC results arc also shown. This example illustrates a typical degree of accuracy variation which occurs as a function of the assumed number of modes. Although damping estimates range from 0.5 to 2.5 percent, the highest confidence is associated with values between 0.5 and 1.0 percent duc to the corresponding high EMAC valucs. MPC values for this mode show only small variations indicating stable mode-shape identification.
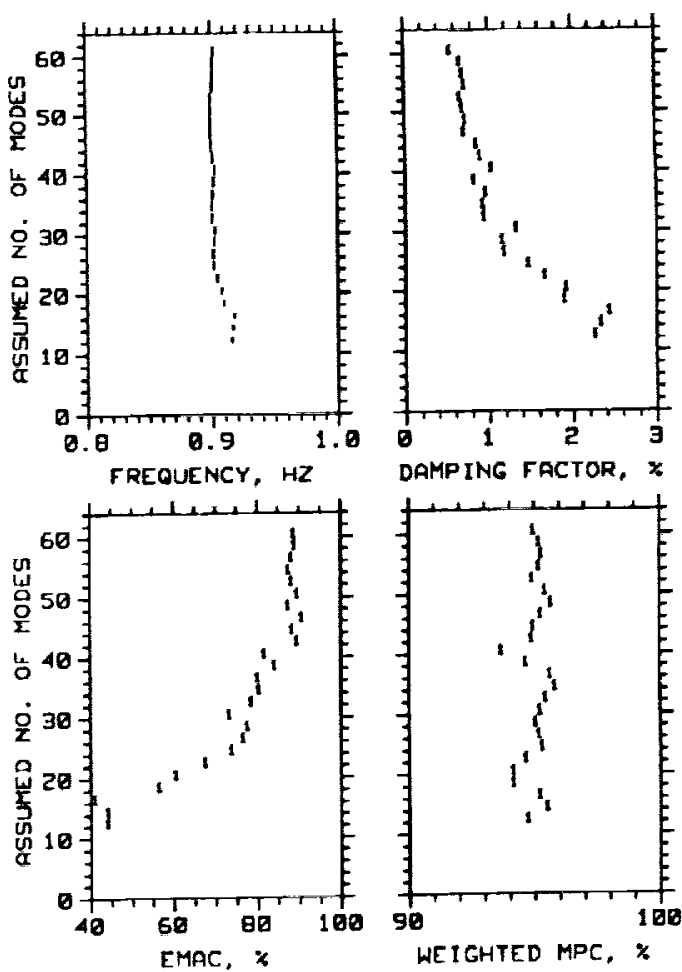

Fig. 4 Identification Results at $0.9 \mathrm{~Hz}$
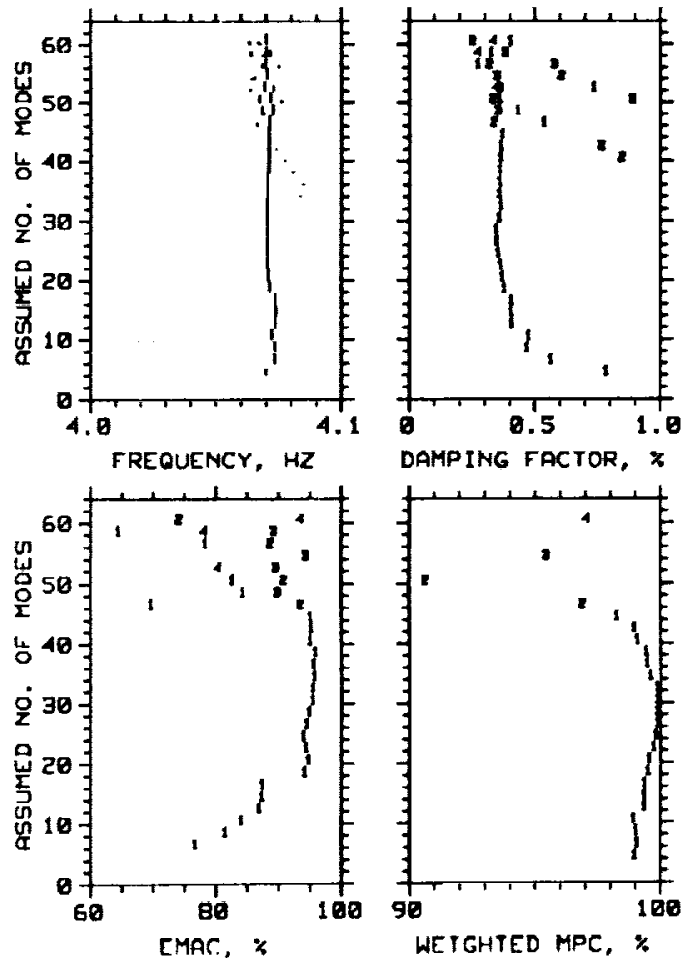

DAMPING FACTOR, $x$

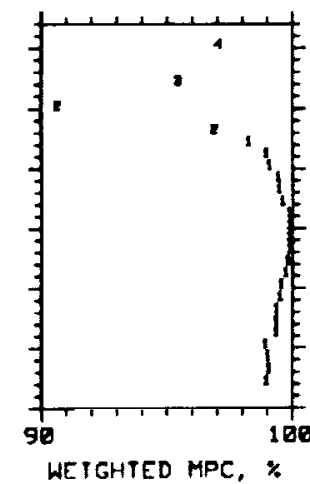

Fig. 5 Identification Results at $4.1 \mathrm{~Hz}$

A more complicated situation is shown in Fig. 5 for the mode (or modes) near $4.1 \mathrm{~Hz}$. The highest EMAC and 
MPC values occur near 30 assumed modes. At higher numbers of assumed modes two additional eigenvalues are identified. These additional results may be weakly excited and/or weakly observed local modes such as the vibration of the rubber air-supply hoses for the thrusters. The important thing to note is that their presence causes significant perturbations in both EMAC and MPC for what is believed to be a single structural mode in this frequency interval. Again, the EMAC and MPC results (and their product, $\mathrm{CMI}$ ) reliably indicate those results with highest confidence based on both temporal and spatial consistency calculations.

Fig. 6 shows a comparison of measured and reconstructed impulse response functions. The ERA analysis used the first 4.5 seconds of measured data. There is only slight difference between the two functions in this interval. Beyond the 4.5-sec data analysis interval, however, the differences between the two functions become considerably larger. Although it is difficult to known the exact reason for the discrepancy between measured and reconstructed responses beyond the data analysis interval, EMAC values in this situation would decrease, highlighting the inconsistency. EMAC quantifies the temporal consistency of each mode beyond the primary data analysis interval on an input-by-input and measurement-by-measurement basis.

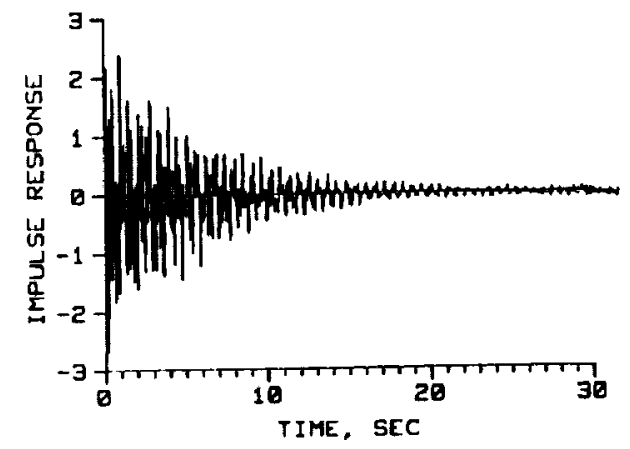

(a) Measured

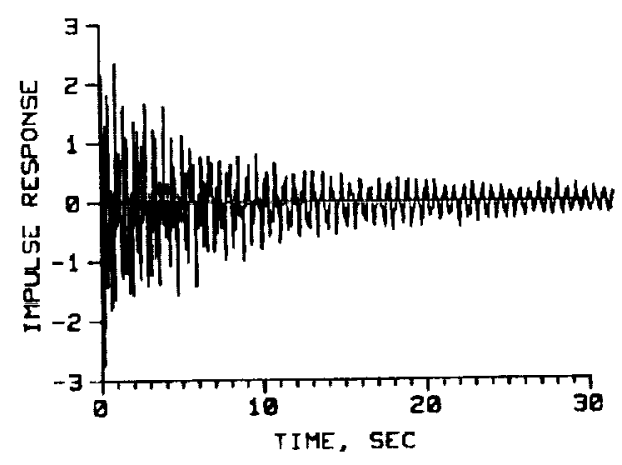

(b) Reconstucted

Fig. 6 Comparison of Measured and Reconstructed Responses
Based on CMI (the product of EMAC and MPC), the best results for the $4.1 \mathrm{~Hz}$ mode (Fig. 5) occurred using 30 assumed modes. The corresponding mode shape is shown in Fig. 7(a). This result is highly believable based on the smoothness and uniformity of motion over the large set of 213 response measurements. Furthermore, this shape and the corresponding frequency are well predicted by a NASTRAN finite element analysis. ${ }^{8}$ Although CMI for this mode is high indicating a reliable result based on consistency calculations, it is also always important to examine the physical deformation pattern of the mode to achieve complete confidence.
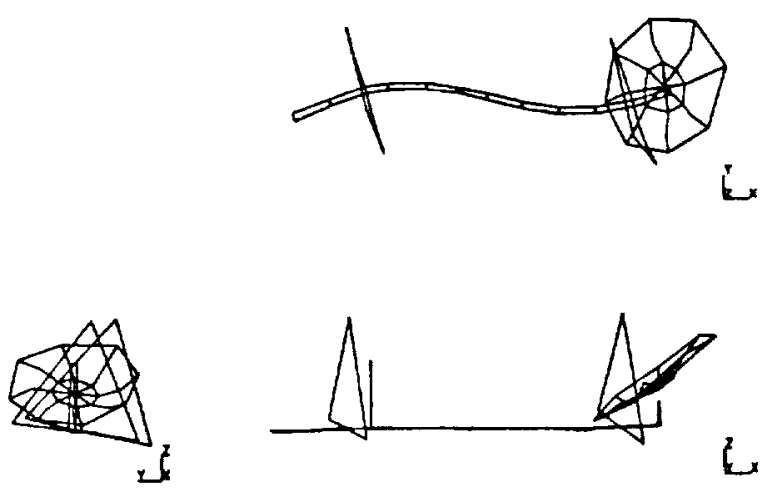

(a) Mode Shape
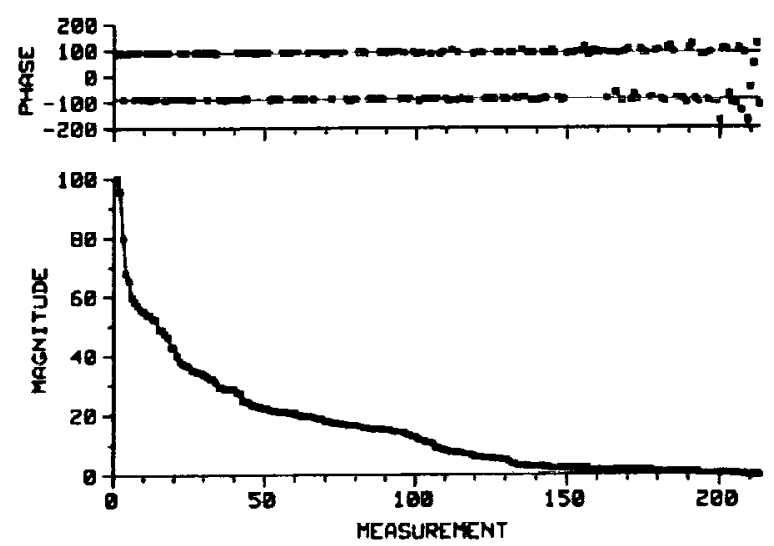

(b) Distribution of Magnitude and Phase Values

Fig. 7 Identified Mode at $4.1 \mathrm{~Hz}$ Using 30 Assumed Modes

To illustrate the rationale for using the standard weighted MP in the CMI calculation rather than the alternative unweighted MPC described earlier, Figure 7(b) shows the distribution of magnitude and phase results for this 4.1 $\mathrm{Hz}$ mode. The values are arranged in ascending order of magnitude. Overall, the identified phase angles cluster near the ideal values of $\pm 90^{\circ}$ and MPC is accordingly high. 
However, a trend of increasing phase angle scatter from $\pm 90^{\circ}$ at smaller magnitudes is seen. These relatively large phase angle errors at small magnitudes are attributed to finite measurement resolution. The overall mode shape is clearly accurate, however, including accurate identification of small magnitudes at appropriate locations (based on Fig. 7a). If unweighted MPC values are used instead of weighted values in the $\mathrm{CMI}$ computation, the $\mathrm{CMI}$ value would be unnecessarily lowered.

It is important to realize that MPC decreases from the ideal value of 100 percent for many reasons other than low response magnitude. For example, significant phase-angle crrors can occur for closely spaced modes which are inadequately uncoupled by excitation. For structures with large numbers of closely spaced modes, such as Mini-Mast which had 108 modes between 14 and $22 \mathrm{~Hz}$ due to the bending of individual truss members ${ }^{7}$, it may be impractical to apply a sufficient number of exciters. Another cause of lower MPC values is the occurrence of true "complex modes" whose eigenvectors are, in fact, significantly non-monophase (i.e., complex) due to a nonproportional damping distribution. Although such situations can occur in practice, low MPC values in most applications are more often the result of identification difficulties, many of which can be eliminated once they are delectod and understood.

\section{Improvement of Results}

With complex structures such as CEM, many modes involve significant motion at only a fraction of the total number of measurement locations. In such circumstances, identification accuracy can be improved by de-emphasizing data with low consistency. Both input and output EMAC values are examined to determine optimum excitation and measurement locations for particular modes based on the results of an initial ERA analysis.

In this application, weighted EMAC values (i.e., EMAC multiplied by the square of the corresponding mode-shape coefficient) are used to select optimum excitation and measurement locations. The analyses use data only for the selected exciters. All response measurements are included in the analysis, however, so that complete mode shape information is obtained. Additional emphasis is achieved for the target mode by retaining only the selected response locations in all block rows of the generalized Hankel matrix below the first $q$ rows, where $q$ is the total number of response measurements. The theoretical basis for permitting the deletion of rows (or columns) of data in the ERA Hankel matrices without affecting the rank of the matrix is well established, 5,12 The approach discussed here is the authors' practical implementation of the theory.

An example of the improvement achieved using this approach is shown in Fig. 8. These results for the $0.9 \mathrm{~Hz}$ mode extend only up to 20 rather than to 60 assumed modes as before (a higher number of assumed modes was unnecessary). Comparing Fig. 8 with Fig. 4 over the range of 2 to 20 assumed modes, significant improvement is observed. EMAC values are appreciably higher and more stable. Corresponding frequency and damping results also show improved stability. Although MPC fluctuates much less than in Fig. 4, the average value of 95 percent is still obtained. This lack of improvement of MPC was unexpected. Normally, such stability is not obtained at MPC values less than 99 percent. The explanation is attributed to the use of piezo-film accelerometers in the experiment. These sensors have considerable phase shift at $0.9 \mathrm{~Hz}$ which is near the lower limit of their operating range. Although all 195 piezo-film sensors were of the same model, appreciable differences in phase response occurred at $0.9 \mathrm{~Hz}$ among the individual units. This anomaly did not occur at higher frequencies.
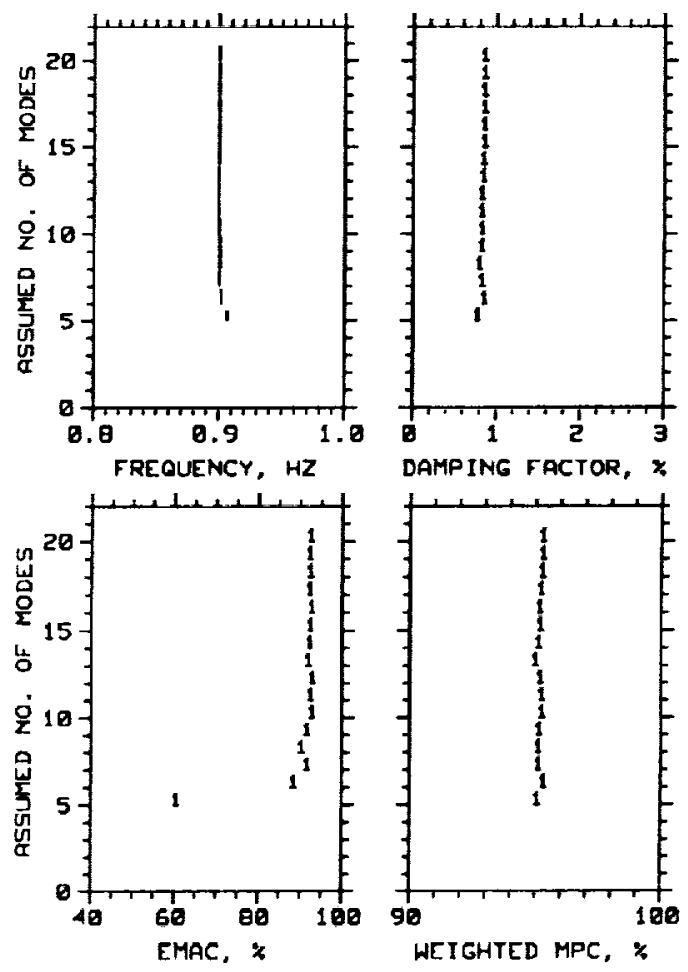

Fig. 8 Improved Identification of $0.9 \mathrm{~Hz}$ Mode 


\section{Conclusions}

The concepts discussed in this paper have been developed over the course of several years in conjunction with many applications of the Eigensystem Realization Algorithm. The result of these efforts is a single parameter known as the Consistent-Mode Indicator which reliably indicates the relative confidence of each identified mode on the basis of both temporal and spatial consistency calculations. In practice, modes with indicator values greater than approximately 80 percent can gencrally be accepted verbatim. Modes with lower values, however, require additional attention. By examining both the Extended Modal Amplitude Coherence, which measures temporal consistency, and the Modal Phase Collinearity, which measures spatial consistency, an explanation for low indicator values can often be developed. Once an explanation is obtained, additional tests and/or data analyses can be performed to improve these results. One approach for improving results of selected modes by deemphasizing data with low consistency was illustrated.

\section{Acknowledgement}

This work was performed in part under a collaborative research agreement between NASA and DLR in the area of Dynamics and Control of Large Space Systems. The modal test of the CSI Evolutionary Model was performed with the assistance of Lockheed Engincering and Sciences Company.

\section{References}

1 Juang, J.-N., Horta, L. G., Phan, M., "System/Observer/Controller Identification Toolbox From Input and Output Measurement Data," NASA Technical Memorandum 107566, February 1992.

2 Niedbal, N. and Hüners, H., Modal-Survey Testing for System Identification and Dynamic Qualification of Spacecraft Structures, AGARD CP-397, Sept. 1985.

3Juang, J.-N. and Pappa, R. S., "A Comparative Overview of Modal Testing and System Identification for Control of Structures, The Shock and Vibration Digest, Vol. 20, No. 6, June 1988, pp. 4-15.

${ }^{4}$ Longman, R. W., Bergmann, M., and Juang, J.-N., "Variance and Bias Confidence Crileria for ERA Modal Parameter Identification," Proc. of AIAA/AAS Astrodynamics Conference, Minneapolis, MN, Aug. 1988, pp. 729-739.
5Juang, J.-N. and Pappa, R. S., "An Eigensystem Realization Algorithm for Modal Parameter Identification and Model Reduction," Journal of Guidance, Control, and Dynamics, Vol. 8, No. 5, Sept.-Oct. 1985, pp. 620-627.

6Pappa, R. S. and Juang, J.-N., "Some Experiences With the Eigensystem Realization Algorithm," Sound and Vibration, Jan. 1988, pp. 30-34.

${ }^{7}$ Pappa, R. S., Schenk, A., and Noll, C., "ERA Modal Identification Experiences With Mini-Mast," NASA Technical Memorandum 4307, Feb. 1992.

$8_{\text {Belvin, W, K., Elliott, K. B., Horta, L. G., Bailey, J. }}$ P., Bruner, A. M., Sulla, J. L. and Won, J., "Langley's CSI Evolutionary Model: Phase 0," NASA Technical Memorandum 104165, November 1991.

${ }^{9}$ Pappa, R. S., Schenk, A., Niedbal, N., and Klusowski, E., "Comparison of Two Dissimilar Modal Identification Techniques," Proc. of the International Forum on Aeroelasticity and Structural Dynamics, Aachen, Germany, June 1991. To appear in the Journal of Guidance, Control, and Dynamics.

${ }^{10}$ Structural Dynamics Research Corp., Test Data Analysis (TDAS) User's Guide, 1991.

11 Brcitbach, E., "A Semi-Automatic Modal Survey Test Technique for Complex Aircraft and Spacecraft Structures," Proc. of 3rd ESRO Testing Symposium, Frascati, Italy, Oct. 1973, pp. 519-528.

12 Juang, J.-N. and Pappa, R. S., "Effects of Noise on Modal Parameters Identified by the Eigensystem Realization Algorithm," Journal of Guidance, Control, and Dynamics, Vol. 9, No. 3, May-June 1986, pp. 294-303. 
Public reporting burden for this ollection of information is estimated to average 1 hesur per response. inrluding the time for reviewing instructions, searching existing data sources fathering and maintaining the data needed. and completing and reviewing the collection of infurmation Swnd comments regarding this burden estimate or any other aspect of this Davis itighway, Sulte 1204. Arlington, VA 22202-4302, and to the Offic of Management and Budget. Paperwork Reduction Project (0704-0 188). Washingron, DC 20503.

\begin{tabular}{|l|l|l}
\hline 1. AGENCY USE ONLY (Leave blank) & $\begin{array}{c}\text { 2. REPOAT DATE } \\
\text { April } 1992\end{array}$ & $\begin{array}{c}\text { 3. REPORT TYPE AND DATES COVERED } \\
\text { Technica1 Memorandum }\end{array}$ \\
\hline
\end{tabular}

\section{TITLE AND SUBTITLE}

A Consistent-Mode Indicator for the Eigensystem Realization Algorithm

6. AUTHOR(S)

Richard S. Pappa, Kenny B. Flliott, and Axel Schenk

\section{PERFORMING ORGANIZATION NAME(S) AND ADDRESS(ES)}

NASA Langley Research Center

Hampton, VA 23665-5225

\section{SPONSORING / MONITORING AGENCY NAME(S) AND ADDRESS(ES)}

National Aeronautics and Space Administration

Washington, DC 20546-0001

\section{FUNDING NUMBERS}

WU 590-14-61-01
8. PERFORMING ORGANIZATION REPORT NUMBER
10. SPONSORING / MONITORING AGENCY REPORT NUMBER

NASA TM- 107607

11. SUPPLEMENTARY NOTES

Pappa: Langley Research Center, Hampton, VA; Elliott: Langley Research Center, Hampton, VA; Schenk: DLR, Germany

To be presented at the 33rd Structures, Structural Dyanmics, and Materials Conference Apri1 13-17, 1992, Dallas, TX

12a. DISTRIBUTION/AVAILABILITY STATEMENT

12b. DISTRIBUTION CODE

Unclassifled-Unltmited

Subject Category 39

\section{ABSTRACT (Maximum 200 words)}

A new method is described for assessing the consistency of structural modal parameters identified with the Eigensystem Realization Algorithm. Identification results show varying consistency in practice due to many sources including high modal density, nonlinearity, and inadequate excitation. Consistency is considered to be a reliable indicator of accuracy. The new method is the culmination of many years of experience in developing a practical implementation of the Eigensystem Realization Algorithm. The effectiveness of the method is 11lustrated using data from NASA Langley's Controls-Structures-Interaction Evolutionary Model.

\begin{tabular}{|c|c|c|}
\hline \multicolumn{3}{|l|}{ 14. SUBJECT TERMS } \\
\hline \multicolumn{3}{|c|}{$\begin{array}{l}\text { Eigensystem Realization Algorithm } \\
\text { Modal Identification } \\
\text { Structural Dynamics Testing }\end{array}$} \\
\hline $\begin{array}{l}\text { 17. SECURITY CLASSIFICATION } \\
\text { OF REPORT }\end{array}$ & $\begin{array}{l}\text { 18. SECURITY CLASSIFICATION } \\
\text { OF THIS PAGE }\end{array}$ & $\begin{array}{l}\text { 19. SECURITY CLASSIFICATION } \\
\text { OF ABSTRACT }\end{array}$ \\
\hline Unclassified & Unclassified & \\
\hline
\end{tabular}

